\title{
Design of Office Automation Information Management System for editorial department
}

\author{
ZHANG Xiaoqian \\ Editorial Office of <Computer Simulation> Beijing 100048
}

\begin{abstract}
Key words: editorial department; office automation; information management system; system design
\end{abstract}
Abstract: In the information age, the information flow of the editorial department is large and the complicated management system has been unable to meet the requirements of the rapid processing of massive information. In order to solve above problems, this paper design an office automation information management system for editorial department. The design of the system mainly consists of hardware system and software system. Wherein the software system designed a large database to facilitate the storage of data, and the database uses a new type of data transmission method through which data can be achieved quickly for the purpose of improving the processing speed of the management system. Through the comparison of the conventional management system and the designed management system, it is proved that the designed system can deal with the data more quickly.

\section{CLC number: G420 Document code:}

With the advent of the information age and the development of communication, there are more and more demands for information processing and management capacity. The traditional management methods and management tools have been difficult to satisfy the modern requirements. The arrival of the information age has led to the growth of data information, only the use of modern management tools and reference to modern management techniques can realize better control of information. Recently, lots of area use computers combining modern information technology in management which gradually transformed into a quantitative, systematic, scientific and automated management model. Such a management approach has become particularly valuable in the days that we need to deal with massive information. This paper has carried on thorough research to the office management system of the editorial department, and the design and the application of the information management system. The automatic information management system researched in this paper can manage and run the information of the editorial department more scientifically and reasonably, ensure the normal operation of the information processing, and help to manage the information more standardized [1].

\section{System Objectives and Analysis}

The information data to be processed by the editorial department on a daily basis mainly includes:

(1) Manage the text of all the manuscripts. Including the real-time entry of the manuscript, the review information of the manuscript, the comments after the review, the progress of the author's revision, the editor's comments on the manuscript, and the editing of the contents of the manuscript.

(2) Record and sort the information of the published manuscript. It includes the article's keywords, release time, release form, author, title and other specific information, but also the manuscript query index and database registration methods.

(3)Arrange and record the opinion and amendment put forwarded by the experts, which includes the research direction of the experts, the content of article that has been commented, the statistics of the actual work and workload of the experts, and optimization and registration of experts' information.

(4) Records and work arrangements of the business carried out by the editorial department. This includes information exchange with other magazine and writing team, recording the content of the inspection and the approval of the work, the subscription amount of various units and the way of subscription. We need to mainly record the exchange of information, and information management is needed for the record approaches and number of records.

(5) Report to the search center, including the record and management of the English content of the published articles. Therefore, as a complete independent automatic information management system, the automation 
information management system for editorial office office should be able to carry out the following processing of the manuscript: the effective handling of the manuscripts, the information processing and exchange for the published manuscripts, the processing of subscription information, the processing of experts reviewed manuscript[2-3]. For the information of the editorial department and the information of the retrieval center, the editorial office automation information management system should have the following function: record, index, query, statistics, modify, edit and so on. Among them, the information statistics for the manuscript is particularly important. It is the effective data affecting the information management of manuscripts in editorial department, the academic level of manuscripts and the degree of approval of manuscripts.

\section{System module structure design}

\subsection{System logic hierarchy}

The office automation information management system for editorial department uses the structured design. The first layer is control module, the second layer is hierarchical control module, and the third layer is operation and function modules. The operation of these layers is independent from each other. You may return to the menu at the end of each operation[4-5]. The communication between modules mainly based on the information of the database. Each layer is independent, which effectively avoids the information conflict, and reduces the operation errors. The hardware module of the system is mainly divided into eight parts, through which can speed up the processing of logarithmic information.

Figure 1 the structure diagram of the system module

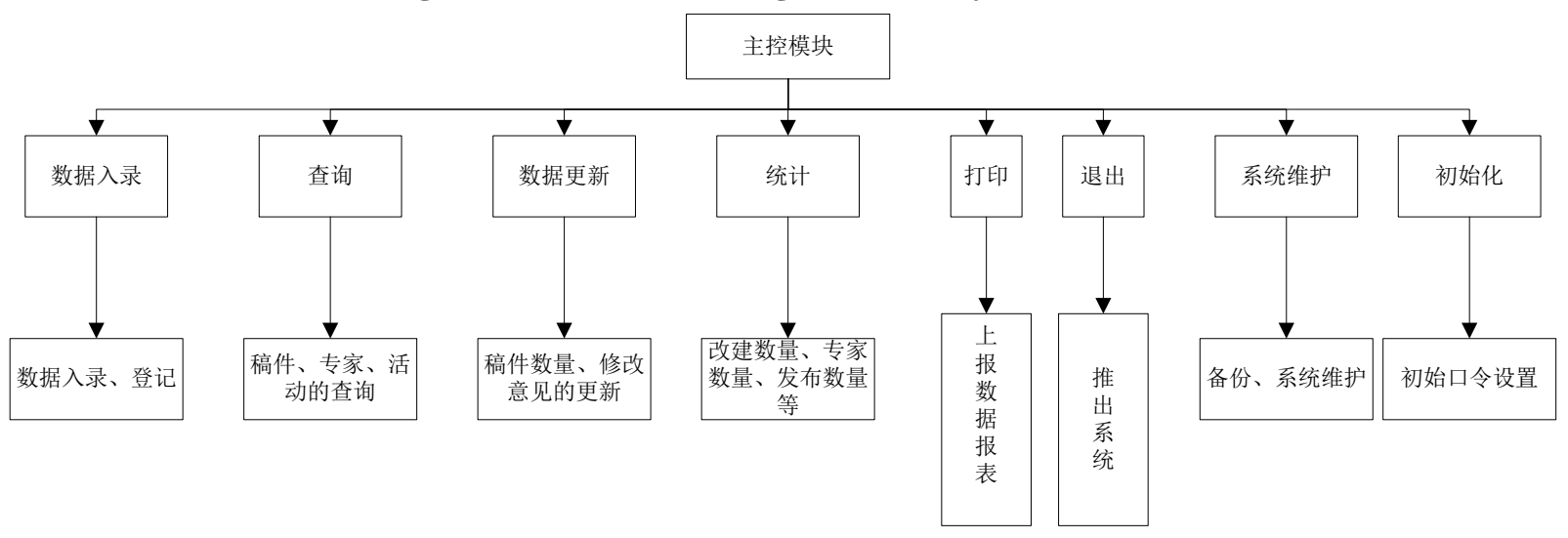

\subsection{Login module}

The login system is mainly responsible for the verification of identity and authority of stuff. The system customizes the setting based on different permissions. The login system effectively protects the system through password and verification code [6-7].

\subsection{Query module}

The role of the query module is to realize in time information query and browse. The query module in the system is designed to query data directly from the database. The system achieves quick query through keywords, number, author name, title and other effective entry of the manuscript.

\subsection{Modify module}

The modify module is used to change, delete, and recover the entry information in the database. Due to the large flow of information, the editorial department needs to set up a modify module to facilitate the modification. The modify module first backs up the data, and then makes changes. Finally, with comparison of the two copies of data, the module realizes the changes.

\subsection{Statistical module}

The statistical module is mainly used for statistics of data in database. First with the help of query module we find the associated data. Once found, the associated data need to be accumulated. When the search is completed, the accumulation ends. Finally, the results are displayed on the screen. Data statistics is achieved through the query module. 


\subsection{Output module}

System output module is mainly on the operation of the content display. The output module is first associated with each module, and then output the operation of each step. The output is displayed on the screen.

\subsection{Initialization module}

System initialization module can be used for the initial system settings according to different forms. The system time saves the backup of the original database while establishing the database. The start of initialization module will activate the replace password of the module. The system uses the original backup data to replace the existing data, and then to achieve the system initialization settings.

\subsection{Maintenance module}

The main purpose of the maintenance module design is to back up and maintain the data in the database. The module can choose to save data. Once the data is lost or damaged due to external causes, the effect of data recovery can be achieved by executing the module. The module is dependent on the database.

\section{System software architecture}

\subsection{Database upgrade om the software system}

The editorial department uses a non-relational database. Because non-relational databases preloads data, it has the ability to quickly access data. Even there is no need to read data, the non-relational database preloads the data for the next quick access. Non-relational database can meet the requirements of high read and write frequency and small read data in the editorial department. In order to avoid too much dependence of the conventional system on the database, non-relational database automatically set the cache time. When the data storage capacity reaches a certain limit, the available data will be automatically stored on the hard disk, unreliable data will be automatically deleted [8-9].

The data of the system database is mainly divided into three parts: text data, graphic data and image data, as shown in the figure 3.

\section{Figure 2 system data composition diagram}

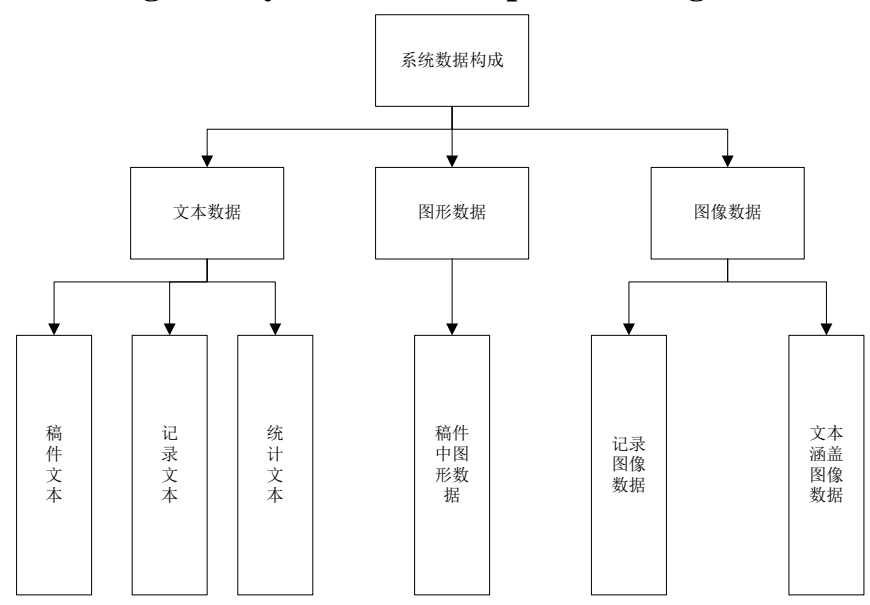

\subsection{Improvement of Transmission Efficiency in Software Design}

As shown in the figure, packets are transferred from route $\mathrm{M}_{1}$ to $\mathrm{M}_{\mathrm{m}}$. Assuming that the time the packet is used to transfer through neighboring nodes is $\Delta \mathrm{t}$, the number of nodes passing through is $\mathrm{n}$, the bandwidth of transmission is $\mathrm{B}$, the size of the transmitted data is $\mathrm{D}$, the size of data contained in the package is d, and the transmission time is $\mathrm{T}$, theoretically the system transmission speed is $\mathrm{S}$ :

$$
\begin{gathered}
\Delta \mathrm{t}=\mathrm{d} / \mathrm{B} \\
T=\frac{D}{B}+\mathrm{n} \Delta \mathrm{t} \\
S=\frac{D}{T}+\frac{D}{\frac{D}{B}+\mathrm{n} \Delta \mathrm{t}}=\frac{D}{\frac{D}{B}+\frac{\mathrm{nd}}{B}}=\frac{1}{\frac{1}{B}+\frac{\mathrm{nd}}{B D}}
\end{gathered}
$$


In the formula, $\mathrm{B}$ is the actual transmission width, $\mathrm{M}$ is the transmission node, and $\mathrm{d}$ is the size of the package transmitted[10]. Wherein the actual transmission width B can be approximated as a constant, so that the theoretical transmission speed $\mathrm{n}$ is related to the reverse of the transmission and is positively correlated with the $\mathrm{D} / \mathrm{d}$. In other words, the smaller the number of M nodes passed, the more data packets transferred, the faster the system transmission speed. So the system reduces the transmission nodes of data packets, while using pressurized form to to speed up the transmission.

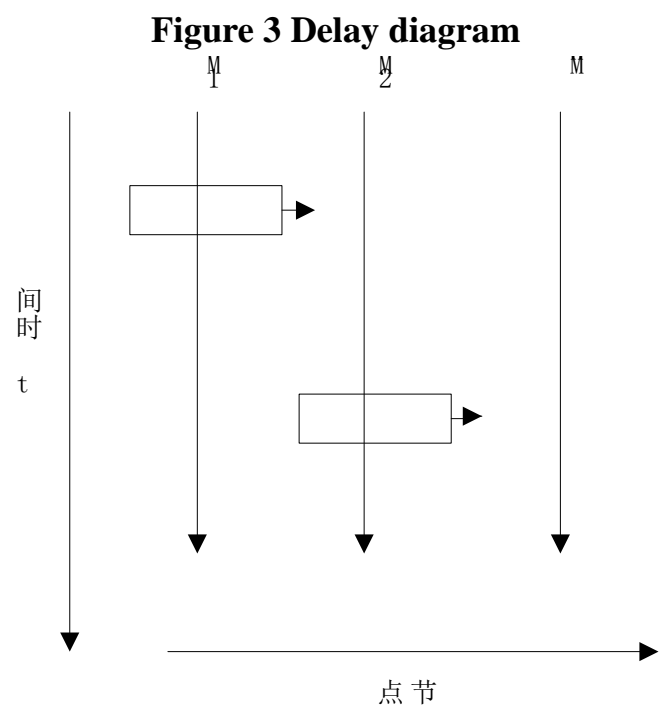

\section{System testing and analysis}

In order to verify the effectiveness of the system, we chose two sets of hardware equipment of the same level. One set used the designed automatic information management system, and the other set used conventional office management system. In a certain period of time, we calculated and compared the storage capacity. As shown in the figure, because the designed system used non-relational database, it's storage speed was much higher than the conventional management system.

Figure 4 Comparison of the amount of information stored in the same time

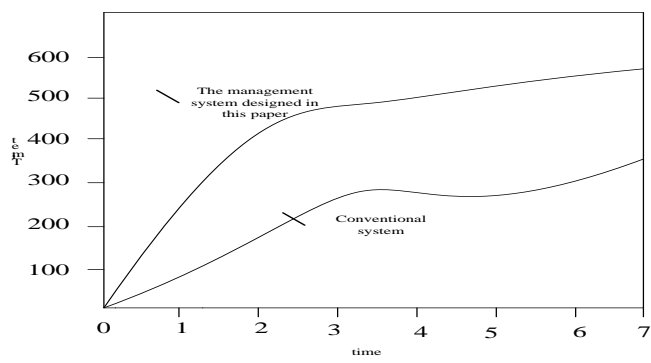

\subsection{High speed read and write performance test}

The designed system reduces the transmission nodes of data packets, while using pressurized form to to speed up the transmission. Same as the first test, we chose two same hardware equipment for the comparison test. One set used the designed automatic information management system, and the other set used conventional office management system. Reading and output the information in database in the same time period, the result is shown in Figure 5. Seven tests were carried out totally. The read and write speed of the conventional management system is about 500 per second, while the speed of the designed system is 700 per second. Through the above test, we can see that the designed system is more efficient in data processing 
Figure 5 The 7 results of the designed system

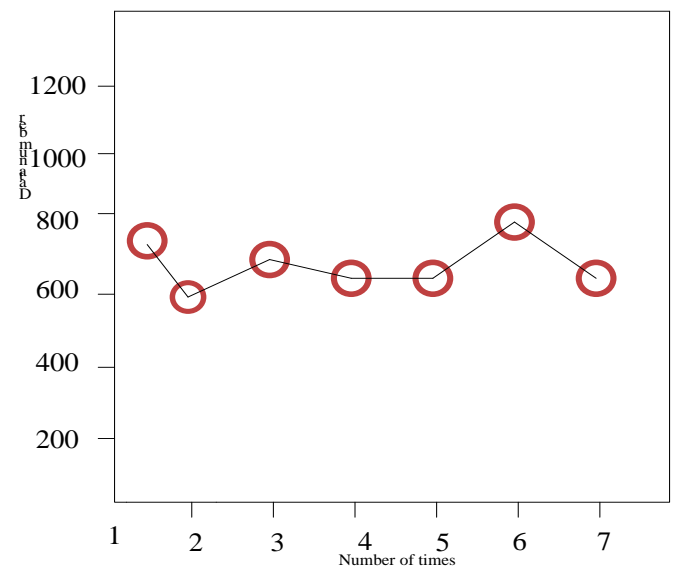

Figure 6 The 7 results of the conventional system

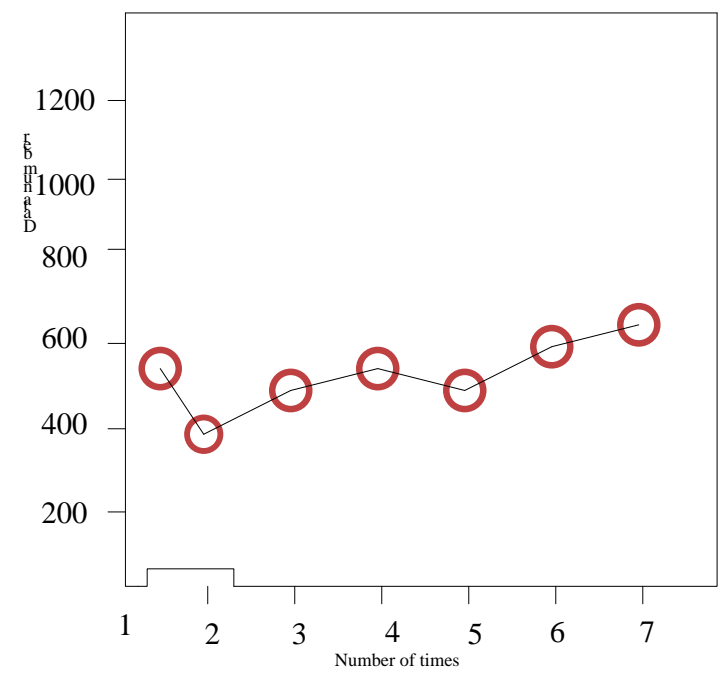

\section{Conclusions}

This paper designs the office automation information management system for the editorial department. The experiment verifies the effectiveness of the system. The management system greatly increases the system's processing capacity by using non-relational database, reducing the transmission nodes of the packet, and using pressurized form in multi-data transmission. It is hoped that this paper can provide some theoretical basis for the editorial office automation information management system.

\section{Reference:}

[1] Campbell M M. The design and use of a data dictionary system for the management of data bases and forms in an office automation system[J]. Journal of Paleontology, 2015, 2(4):1113-1116.

[2] Wang S, Li D, Wang W, et al. Research and Implementation on Integrated Automation Equipment and Information Management System Over Whole Province[J]. Electrical Engineering, 2016.

[3] Waskitho N T, Djudiah. Controlling Model of Intangible Assets on Secondary Irrigation System Management[J]. Quarterly Journal of the Royal Meteorological Society, 2016, 48(204):365-370.

[4] Xiong G, Shen D, Dong X, et al. Parallel Transportation Management and Control System for Subways[J]. IEEE Transactions on Intelligent Transportation Systems, 2016, PP(99):1-6.

[5] Jiang Y, Liu C C, Diedesch M, et al. Outage Management of Distribution Systems Incorporating Information From Smart Meters[J]. IEEE Transactions on Power Systems, 2016, 31(5):4144-4154.

[6] Pandey C, Khobragade P, Jain A, et al. Management Information Systems (HMIS): A Method for 
Strengthening HMIS in Uttar Pradesh, India.[J]. Lancet, 2015, 2(7716):605.

[7] Lee Y T, Hsiao W H, Huang C M, et al. An integrated cloud-based smart home management system with community hierarchy[J]. IEEE Transactions on Consumer Electronics, 2016, 62(1):1-9.

[8]Libin Z, Zhang X, Zhiyong L U, et al. Design and Implementation of the Mapping Data and Information Management System[J]. Canadian Journal of Cardiology, 2015, 31(10):2012-2018 vol.3.

[9] Ueda T, Masuko S, Araoka F, et al. Development of a Model for Successful Implementation of Supply Chain Management Information System in Indian Automotive Industry[J]. Journal of Neuroscience Research, 2015, 19(3):248-262.

[10] Young S L, Pitla S K, Van Evert F K, et al. Moving integrated weed management from low level to a truly integrated and highly specific weed management system using advanced technologies[J]. Weed Research, 2017, 57(1):1-5. 